\title{
Factores psicosociales y
portamiento agresivo al conducir \\ Factores psicosociales y
comportamiento agresivo al conducir correlacionados con accidentes de tránsito en conductores salvadoreños
}

\author{
Psychosocial factors and aggressive driving behavior in reciprocity \\ to traffic accidents caused by salvadorean drivers
}

\author{
José Ricardo Gutiérrez-Quintanilla' \\ jose.gutierrez@utec.edu.sv \\ Marlon Elías Lobos-Rivera² \\ Universidad Tecnológica de El Salvador
}

\section{Resumen}

El presente estudio tiene como objetivo analizar si los factores psicosociales y el comportamiento agresivo al conducir están relacionados con los accidentes de tránsito de los conductores. El estudio es de tipo ex post facto, con diseños retrospectivo y transversal, con un muestreo no probabilístico de tipo intencionado. La muestra estuvo conformada por 1.012 conductores del transporte público y privado, de ambos sexos. La técnica fue la encuesta. La media de edad total fue de 34.81 años, con una DT (Desviación Típica) de 11.02. En hombres, la media de edad fue de 34.84 años, con una DT de 11.15; y en las mujeres, de 34.65 años, con una DT de 10.29. Entre las variables sociodemográficas están las siguientes: 152 (15,0 \%) son mujeres y 860 (85,0 \%) hombres; según los años de experiencia de conducir: los grupos de 1 a 5 años, 341 (33,9\%); de 6 a 15 años, 379 (37,7\%); de más de 16 años, 285 (28,4\%). Los instrumentos utilizados fueron: Escala de bienestar laboral, Escala Magallanes de estrés, Cuestionario de salud general, Escala de ansiedad y depresión, "Inventario de expresión de la ira

\section{Abstract}

The objective of this study is to analyze whether the psychosocial factors and the aggressive behavior of drivers in El Salvador are related to the car accidents they get involved in. This is an ex post facto study, with transversal and retrospective designs; an unintentional, non-probabilistic sampling was used. The sample included 1,012 private and public drivers; both men and women were involved in the study. A survey was conducted with the drivers. The overall median age was 34.81 years, with a TD (Typical Deviation) of 11.02. The median age of men was 34.84 with a TD of 11.15 , and the median age of women 34.65 with a TD of 10.29 . The sociodemographic variables included the following: $152(15,0 \%)$ were women and $860(85,0 \%)$ were men. According to the number of years of experience, they were divided as follows: 1-5 yrs. of experience, 341 (33,9\%); 6-15 yrs. of experience, 379 $(37,7 \%)$; more than $16 \mathrm{yrs}$. of experience, $285(28,4 \%)$. The instruments used included the general work wellbeing questionnaire (QBLG), the Magallanes scale of stress (EMEST, given its Spanish Acronym), the Goldberg

José Ricardo Gutiérrez-Quintanilla. Es Doctor en Psicología. Profesor titular e investigador. Universidad Tecnológica de El Salvador.

2 Marlon Elías Lobos-Rivera. Es Licenciado en Psicología. Coinvestigador. Universidad Tecnológica de El Salvador. marlon.lobos28@gmail.com 
del conductor" y Driving Log. En los resultados se encontró que existe una correlación importante entre los factores psicosociales y el comportamiento agresivo al conducir con los accidentes de tránsito cometidos.

\section{Palabras clave}

Agresividad (psicología); Conducta (psicología); accidentes de tránsito - Investigaciones- El Salvador; Seguridad vial El Salvador

\section{Introducción}

Los accidentes de tránsito tienen implicaciones muy graves, tanto para las víctimas como para el Estado y para los mismos conductores. El comportamiento agresivo al conducir podría estar relacionado con la personalidad, la psicopatología, el estrés laboral, la educación vial, la señalización de las carreteras, la saturación vehicular, la distracción al volante, entre otras causas. Según datos del Fondo de Prevención Vial (2003), en este aspecto El Salvador se ubicó en el primer lugar en Latinoamérica y el Caribe con una tasa de 23.70 muertos por cada 100 mil habitantes por accidentes de tránsito. Asimismo, ocurren más de 25 mil accidentes de tránsito cada año (PNC, 2007). De acuerdo con las estadísticas de la Subdirección de Tránsito de la Policía Nacional Civil (PNC), el año 2013 cerró con 1.021 fallecidos en accidentes de tránsito (23 más que en 2012), 9.434 lesionados y 23.098 percances viales.

La mayoría de las víctimas recibieron atención medica en los diferentes establecimientos del sistema público de salud (PNC, 2013). Del total de decesos, 362 ocurrieron en hospitales, de estos 292 eran hombres y 70 mujeres, que representan el $9 \%$ de los lesionados. Se reportaron 4.123 hospitalizaciones (3.023 hombres y 1.100 mujeres) por accidentes de tránsito. Mientras que en 2012 ocurrieron 397 muertes y se reportaron 4.359 egresos hospitalarios. El mayor incremento se dio en las emergencias por accidentes de tránsito, que subieron en el año 2013. Solamente emergencias de tránsito, sin tomar en cuenta otras consultas, pasaron de 8.857 a 10.893 (Ruales, 2014). El promedio de los costos de egreso de emergencia fue de $\$ 1.513 .07$ por persona atendida; el de consulta de emergencia fue de $\$ 75.71$ por paciente atendido, y el costo de la consulta externa ambulatoria es de \$35.03. En suma, el sistema de salud pública invirtió \$7.557.777.87 en atención de víctimas de accidentes de scale of anxiety and depression, the "Driver Anger Scale" and the Driving Log. The findings showed that there is a relevant reciprocity between the psychosocial factors and aggressive driving behavior in relation to traffic accidents.

\section{Keywords}

Aggressiveness (psychology); Behavior (psychology); car accidents - Research- El Salvador; Traffic Safety El Salvador.

tránsito el año 2013; y el año 2012 fue de \$7.709.865.70 (Minsal, 2016). En la actualidad los costos que el Estado tiene en atender las emergencias, las hospitalizaciones y la consulta ambulatoria de lesionados por accidentes de tránsito rondan los 8 millones de dólares anuales (Minsal, 2016). Sin embargo, hay que destacar que existen emergencias que son atendidas en el sistema privado de salud, lo cual no se refleja en estos datos. También existen otros costos que no son cuantificados en este estudio, como los daños materiales de los vehículos causados a la propiedad privada y las pérdidas económicas familiares, estatales y empresariales por los días laborales perdidos debido a las incapacidades y discapacidades sufridas por las víctimas en los accidentes de tráfico.

Al adoptar una meta sobre los traumatismos provocados por los accidentes de tránsito, también se reconoce la sólida base empírica que existe en lo que respecta a las medidas que funcionan para reducir los accidentes de tránsito. Se dispone de una gran cantidad de datos que permiten saber cuáles son las intervenciones más eficaces para mejorar la seguridad vial. Los países que las han llevado a cabo con éxito lograron reducir el número de víctimas mortales en carretera. La Asamblea General de las Naciones Unidas pidió a la Organización Mundial de la Salud (OMS) que hiciera un seguimiento a los progresos realizados por medio de su serie titulada "Informe sobre la situación mundial de la seguridad vial". La reducción de las muertes y traumatismos por accidente de tránsito en un $50 \%$ para 2020 está incluida entre las metas de los Objetivos de Desarrollo Sostenible (OMS, 2015). El número de muertes por accidente de tránsito a escala mundial fue de 1.25 millones en 2013. El problema se está estabilizando, aún con el aumento mundial de la población y del uso de vehículos de motor. Entre 2010 y 2013, la población ha aumentado en un 4 \% y los vehículos en un $16 \%$, lo cual indica que las intervenciones puestas 
en práctica en los últimos años para mejorar la seguridad vial en el mundo han salvado vidas humanas. Las tasas de mortalidad por esta causa en los países de ingresos bajos ascienden a más del doble de las registradas en los países de ingresos altos; y hay un número desproporcionado de muertes en relación con su nivel de motorización: el 90 \% de las muertes por accidentes de tránsito se producen en países de ingresos bajos y medios, aunque esos países concentran el 54 \% de los vehículos del mundo (OMS, 2015).

Desde la perspectiva biológica, la conducta agresiva en sus distintas modalidades sirve a la conservación individual y de la especie, supone la interacción entre la producción hormonal y la acción de determinados neurotransmisores, inhibidores o facilitadores. Sin embargo, la manifestación de la conducta agresiva va a depender de factores de distinta naturaleza y de gran complejidad, sobre todo en los seres humanos; entre aquellos la experiencia previa, la existencia de estados internos predisponentes y los determinantes situacionales. En el desencadenamiento y desarrollo de todas las conductas motivadas, y el de la agresividad, por más fuerte que parezca su carácter biológico, juegan un papel importante la estimulación externa, la anticipación de posibles necesidades y la intervención de la experiencia o la historia de aprendizajes previos. La agresividad también surge por deficiencias en la regulación del sistema emocional, adoptando la forma de una excesiva manifestación expresiva; pero traducida en conductas intencionales puede llegar, en forma manifiesta o encubierta, a intentar perjudicar o infligir daño (físico, moral, psíquico y social) a sí mismo o a otra persona, e incluso contra objetos inanimados, como símbolos o enseres. Los psicólogos han argumentado sobre la naturaleza de la agresión. Para algunos, la agresión es una conducta innata; otros argumentan que la agresión constituye una respuesta a la frustración, que no es un instinto independiente; que la conducta agresiva es aprendida, no innata. Pero también se dan por alteraciones de la personalidad y como resultado de lesiones de algunas áreas específicas del cerebro, como la amígdala, el hipocampo y el lóbulo frontal.

Los factores psicosociales en el trabajo son complejos y difíciles de entender, debido a que representan el conjunto de percepciones y experiencias del trabajador (OIT, 1986). Es relevante diferenciar entre los factores psicosociales y los factores psicosociales de riesgo. Los primeros son descripciones que se refieren a la estructura organizacional (Minztberg, 1993); a las condiciones psicosociales del trabajo, como la cultura corporativa, el clima laboral, el estilo de liderazgo, el diseño del puesto de trabajo, factores que como tales pueden ser positivos o negativos (Kalimo, El-Batawi, \& Cooper, 1988). Los segundos son predictivos, se refieren a las condiciones laborales que tienen la probabilidad de producir efectos lesivos a la salud de los trabajadores, son elementos que pueden afectar negativamente la salud y el bienestar del trabajador (Benavides et al., 2002), son factores desencadenantes de tensión y estrés laboral.

Los factores psicosociales de riesgo son factores probables de daño a la salud, son negativos y pueden afectar tanto a la salud física como la psicológica (Cox, \& Griffiths, 1996), son factores de estrés que pueden alterar y desequilibrar los recursos y las capacidades de la persona para manejar y responder al flujo de la actividad derivada del trabajo (Taris, \& Kompier, 2005). Pueden provenir de los múltiples componentes del trabajo: falta de control, dedicarle muchas horas, intensidad de su ritmo de trabajo, horarios cambiantes e imprevisibles, mala comunicación, ambigüedad o sobrecarga del papel laboral. Los principales modelos de estrés laboral (Cooper, 1998; Cooper, 2001) han expuesto los mecanismos principales que dan lugar a los factores de estrés laboral; y la investigación psicofisiológica, médica y psicológica ha propuesto diferentes tipos de procesos que relacionan los factores de estrés laboral con el daño a la salud física y mental (Selye, 1975; Lazarus, \& Folkman, 1986; Hobfoll, 1989; McEwen, 2002).

En el presente estudio de los conductores salvadoreños, se hace referencia a los factores psicosociales de riesgo al conducir. En este sentido, se han definido y señalado variables importantes como el bienestar laboral, estrés general, bienestar psicológico, funcionamiento social, salud mental, ansiedad y depresión, que pueden estar relacionadas con el comportamiento agresivo al conducir y en conjunto incidir en los accidentes de tránsito cometidos por los conductores salvadoreños. Los factores psicosociales de riesgo amenazan la salud de las personas, tienen características propias que hacen más difícil su manejo, evaluación y control. Entre las características más importantes podrían citarse las siguientes: se extienden en el espacio y el tiempo, son difíciles de objetivar, afectan a los otros riesgos, tienen escasa cobertura legal, están moderados por otros factores y son difíciles de modificar. Estas características dificultan su prevención, evaluación y control. Los factores psicosociales de riesgo laboral son situaciones laborales que tienen una alta probabilidad de dañar gravemente la salud de las personas, física 
y mentalmente. Los factores psicosociales de riesgo laboral son situaciones que afectan habitualmente de forma importante y grave la salud. Mientras que los factores de riesgo psicosocial tienen habitualmente diferentes niveles de probabilidad de ocasionar daños de todo tipo. Los factores psicosociales de riesgo tienen una alta probabilidad de generar consecuencias principalmente graves para las personas.

Galovski y Blanchard (2002) demostraron que los conductores agresivos presentan mayores niveles de estrés general y ansiedad. También expresan que el origen de los pensamientos agresivos se debe al uso del automóvil y que se manifiestan en actuaciones agresivas. Parry (1968) plantea que los automóviles originan pensamientos agresivos en los conductores y los incitan a provocar conductas agresivas. Por otra parte, se ha evidenciado que los hombres son más agresivos físicamente que las mujeres (Archer, Kilpatrick \& Bromwell, 1995; Bettencourt \& Miller, 1996). Sin embargo, investigaciones que comparan el género en función de la agresión vial fueron menos concluyentes. Si bien es cierto que los hombres informaron mayores niveles de agresión vial, no se demostraron diferencias significativas en la conducción agresiva en relación con las mujeres. También se observa que los conductores del transporte público (autobuses y microbuses) y particulares adoptan actitudes prepotentes y agresivas, por conducir un vehículo de mayor tamaño, hacia los conductores de vehículos pequeños. En estos casos, tienen sentido las características de personalidad (Dahlen, Martin, Ragan \& Kuhlman, 2005), ya que están relacionadas con la conducción vial. Estos autores encontraron relación entre las variables como la cólera, impulsividad, agresividad general y agresión vial. Aunque la mayoría de los estudios tienden a usar muestras no forenses, la agresividad del conductor y el furor vial reciben atención pública debido a su naturaleza extrema, y se pueden observar en las poblaciones que demuestran disposiciones antisociales similares a otros contextos criminales (Junger, West \& Timman, 2005).

Los agresores en la conducción están más propensos a experimentar niveles elevados de cólera en comparación con la población en general. Existen otros factores que pueden ser motivo para generar agresión en los conductores, como el territorio, conocido comúnmente como la ruta que tiene que recorrer el conductor, el espacio personal y el anonimato (Ward, Waterman \& Joint, 1998). Existen algunos conductores enojados que solo expresan la cólera cuando conducen (Lawton \& Nutter, 2002), aunque se considera que la agresividad puede ser un aprendizaje social, donde el indivi- duo ha aprendido y practicado algunos niveles de violencia en su vida. La cólera o enojo en la conducción ha sido considerada como un pronosticador significativo de la agresión vial (Miles \& Johnson, 2003; Ulleberg, 2001). Con referencia al trastorno de personalidad antisocial y el abuso de sustancias, estos constituyen factores de riesgo que aumenta la probabilidad de morir en la carretera. Galovski y Blanchard (2001) compararon motoristas agresivos y no agresivos, y no encontraron diferencias entre cólera vial, hostilidad psicológica y conducta tipo A, pero sí entre ansiedad, hostilidad y cólera general. Los conductores que presentan mayor hostilidad son los que ocasionan más accidentes de tránsito, cometen más infracciones a las normas de tránsito, determinándose que la existencia de un alto grado colérico en estos conductores les originan problemas personales (Deffenbacher, Deffenbacher, Lynch \& Oetting, 2003).

Los conductores que se ven involucrados en altercados con otros conductores reportan más choques y más agresiones de tráfico que el resto de conductores (Hemenway \& Solnick, 1993). Por cada acto de agresión seria, hay miles de conductores enojados, cuyo comportamiento puede poner en peligro su vida y la de otros. El centro o corazón del problema del conductor enojado está en su alto nivel emocional, fisiológico y de su arousal (irradiación de la excitación cerebral). Estas personas son fisiológicamente activos, enojados, furiosos, muy enfadados y se comportan agresivamente (Deffenbacher, Huff, Lynch, Oetting \& Salvatore, 2000; Deffenbacher, et al., 2003; Deffenbacher, Filetti, Richards, Lynch \& Oetting, 2003; Deffenbacher, Lynch, Oetting \& Yingling, 2001; Deffenbacher, Oetting \& Lynch, 1994). Por otra parte, estudios en Gran Bretaña han demostrado que el rasgo de cólera (característica de personalidad) en la conducción correlaciona positivamente con las infracciones de tránsito; y estas están asociadas tanto con incidentes agresivos como con los poco agresivos (Lajunen, Parker, \& Stradling, 1998). El nivel de cólera no es la única variable importante para comprender la relación y las consecuencias negativas del comportamiento en la agresión y la conducción riesgosa; también, tanto el estrés de las personas, la forma de afrontar la cólera como su expresión y su intensidad pueden jugar un papel central en la seguridad y la salud del conductor.

Krahé y Fenske (2002) plantean el papel de la personalidad machista y la edad y el poder (tamaño) del vehículo como pronosticadores de comportamiento agresivo en la conducción. El análisis de regresión múltiple demostró que cada 
uno de los pronosticadores estaban significativamente relacionados con la conducción agresiva: Ios conductores de menor edad, los conductores de autos de mayor potencia y la personalidad machista presentaron significativamente mayor probabilidad de conducción agresiva.

\section{Método}

\section{Participantes}

En el presente estudio de tipo ex post facto (Montero \& León, 2007), se aplicaron los diseños retrospectivo y transversal, se utilizó un muestreo no probabilístico de tipo intencionado en conductores públicos y particulares, la muestra fue de 1.012 conductores. La técnica de recolección de datos fue la encuesta. La media de edad total es de 34.81 años, con una DT de 11.02. En los hombres, la media de edad es de 34.84 años, con DT de 11.15; y en las mujeres, de 34.65 años, con DT de 10.29. Referente a las variables sociodemográficas, $152(15,0 \%)$ son mujeres y $860(85,0 \%)$ hombres. De acuerdo con los años de conducir, se tienen los grupos de 1 a 5 años 341 (33,9\%); de 6 a 15 años, 379 (37,7\%); de más de 16 años, 285 (28,4\%). En cuanto al tipo de vehículo que las personas conducen, se tiene que $131(13,0 \%)$ conducen autobús, 205 (20,3\%) microbús, 478 (47,3\%) vehículo particular y 196 (19,4\%) otro tipo de vehículo (taxi, pick up, rastra y motocicleta). Con respecto a si el conductor sufrió un accidente, 124 (12,3\%) sí han sufrido un accidente, y 886 $(87,7 \%)$ no han tenido accidente; y en relación con el número de veces que presentó enojo en la semana, $435(76,4 \%)$ se enojan de 1 a 3 veces por semana, $93(16,3 \%)$ de 4 a 6 veces, y 41 (7,2\%) más de 7 veces por semana.

\section{Medidas e instrumentos de medición}

Cuestionario de elaboración propia. En la primera página de la batería de pruebas se incluyó un conjunto de variables sociodemográficas, tales como la edad, el estado civil, la residencia, los años de experiencia, el tipo de vehículo que conduce, si hay consumo de bebidas alcohólicas, el número de enojos por semana, entre otras.

Escala Magallanes del Estrés, EMEST (García-Pérez, MagazLago, García-Campuzano, Sandín-Iñigo \& Ceregido-Senín, 2011). Está prueba evalúa alteraciones fisiológicas comúnmente aceptadas como "reacciones al estrés". Se define el estrés como la tensión del organismo, que se ve obligado a movilizar sus fuerzas, sus energías, para defenderse de una situación de riesgo, de peligro, para proteger su integridad biológica. El instrumento consta de quince reactivos, a los cuales la persona debe contestar sobre la frecuencia con que le han sucedido (durante los últimos dos meses) cada uno de los acontecimientos que se expresan. Las opciones de respuesta son cuatro: nunca, a veces, a menudo y siempre. El índice de consistencia interna mediante alfa de Cronbach obtenido en España fue de 0.82. Para el presente estudio se obtuvo un índice de fiabilidad mediante el análisis de consistencia interna alfa de Cronbach de .78

Cuestionario de salud general, GHQ-12 (Goldberg \& Blackwell, 1970). Este es un instrumento de filtrado o tamizaje de síntomas de salud mental o bienestar psicológico. La prueba mide dos factores: Bienestar psicológico (salud mental), que se compone de seis ítems $(1,2,5,7,9,12) ;$ Funcionamiento social, que se compone de seis ítems $(3,4,6,8$, $10,11)$. Estas dos subescalas permiten detectar la presencia y ausencia de síntomas de salud mental. La salud mental se puede definir como un estado de bienestar en el cual los individuos reconocen sus habilidades, son capaces de enfrentarse con los estresantes normales de la vida, pueden trabajar de una manera productiva y fructífera y hacer contribuciones en sus comunidades (Okasha, 2005). La prueba posee adecuadas propiedades psicométricas de fiabilidad y validez. Para el presente estudio, la dimensión bienestar psicológico obtuvo un índice de consistencia interna alfa de Cronbach de .74; y para la dimensión funcionamiento social, obtuvo un índice de consistencia interna de .76. La sumatoria de los ítems de las subescalas de bienestar psicológico y funcionamiento social brindan una escala total de salud mental, la cual obtuvo un índice de consistencia interna alfa de Cronbach de .85, indicando que la prueba posee adecuadas propiedades métricas, en El Salvador, para medir estos problemas psíquicos.

Escala de ansiedad y depresión EADG (Goldberg, Bridges, Duncan-Jones \& Grayson, 1988). Fue desarrollada en 1988 a partir de una versión modificada de la Psychiatric Assessment Schedule, con la finalidad de lograr una entrevista de corta duración, para ser utilizada por médicos no psiquiatras como instrumento de cribado. La versión en castellano ha sido validada por Montón et al. (1993); consta de dos subescalas: una de ansiedad (ítems del 1 al 9) y la otra de depresión (ítems del 10 al 18), con 9 ítems cada una. Todos los ítems son de respuesta dicotómica (Sí, No) en la prueba original y la adaptación española; se da una puntuación independiente para cada escala, con un punto para cada res- 
puesta afirmativa. La ansiedad es definida como una reacción emocional ante la percepción de un peligro o amenaza, manifestándose mediante un conjunto de respuestas agrupadas en tres sistemas: cognitivo o subjetivo, fisiológico o corporal, y motor, pudiendo actuar con cierta independencia (Miguel-Tobal, 1996). La depresión es definida como un trastorno emocional que altera el funcionamiento cognitivo $\mathrm{y}$ las emociones (Beck, 1970). En el presente estudio, los índices de fiabilidad mediante el análisis de consistencia interna alfa de Cronbach fueron, para la subescala ansiedad fue de .82 , y para la subescala depresión de .82 .

Inventario de Expresión de la Ira del Conductor (Driving Anger Expresión Inventory, DAX) (Deffenbacher et al., 2002). Evalúa cuatro formas diferentes de expresar la ira al conducir y está compuesto por 49 ítems contestados en una escala de cuatro puntos: desde 1 (casi nunca) hasta 4 (casi siempre). Recoge cuatro dimensiones: Expresión verbal agresiva, que evalúa la expresión verbal agresiva de la ira mediante expresiones como gritar a un conductor o peatón, miradas furiosas; Expresión física personal agresiva, que evalúa la utilización del cuerpo para expresar agresividad; Uso del vehículo para expresar la ira, que evalúa la tendencia de la persona a utilizar el vehículo como medio de expresión agresiva de la ira; y Expresión adaptaiva-constructiva, que mide el afrontamiento de la ira mediante de comportamientos seguros o psicosociales. La suma total de ítems de las subescalas permiten construir una dimensión general Ilamada Comportamiento agresivo al conducir. El instrumento cuenta con índices de fiabilidad propios. En las subescalas se obtuvieron los siguientes índices: Expresión verbal agresiva, .82; Expresión física agresiva, .77; Uso del vehículo para expresar ira, .75; Expresión adaptativa-constructiva, .91. Para el presente estudio, en las dimensiones referidas, se obtuvieron los siguientes índices de fiabilidad mediante el alfa de Cronbach: Expresión verbal agresiva (ítems 5, 6, 9, 11, 14, 28, 31, 37, 38, 39, 40, 43), .85; Expresión física agresiva (ítems 1, 8, 10, 12, 13, 17, 18, 20, 21, 34, 41), .82; Uso del vehículo para expresar ira (ítems 2, 3, 4, 7, 15, 16, 19 , $22,27,33,46), .81$; Expresión adaptativa-constructiva (ítems $23,24,25,26,29,30,32,35,36,42,44,45,47,48,49)$, .87, indicando que la prueba posee adecuados índices métricos para estudiar estas variables en El Salvador.

Driving $\log (D L)$. Es un cuestionario autoinformado, diseñado y validado por Deffenbacher et al. (2000) y Deffenbacher et al. (2001). En el presente estudio se aplicó la versión del DL traducida del original al castellano por Herrero-Fernández,
Fonseca-Baeza y Pla-Sancho (2014), quienes hicieron el estudio psicométrico (validez y fiabilidad) de adaptación. Este instrumento consta de dos partes, recogiendo información en relación con el día anterior al que la persona contesta el cuestionario. En la primera parte interroga acerca del número de veces que condujo la persona, de kilómetros que realizó y de veces que experimentó ira. La segunda parte consta de 21 ítems, a los que el participante contesta Sí (1) o No (0) en función de si realizó lo que se enuncia en el ítem correspondiente, o no.

Esta parte tiene dos dimensiones, que fueron confirmadas en el estudio de adaptación al castellano: conducción agresiva (ítems $2,4,6,8,10,12,14,16$ ) y conducción arriesgada (ítems 1, 3, 5, 7, 9, 11, 13, 15, 17, 18, 19, 20, 21). Los índices de consistencia interna mediante alfa de Cronbach fueron, para la primera subescala, .73; y para la segunda, .72. En el presente estudio se incorporaron algunos pequeños cambios para mejorar algunos detalles, como los siguientes: en vez de una escala de medida dicotómica (Sí o No), se agregó una de intervalo de 4 opciones de respuesta $(1=$ nunca, $2=$ a veces, $3=$ a menudo y $4=$ siempre). También se incorporaron 6 reactivos para medir distracción al conducir (ítems del 1 al 6, seguidos por los 21 ítems de la prueba original) con el mismo formato de respuesta. La conducción agresiva se define como una serie de acciones cometidas por automovilistas y que son motivadas por disputas con otros conductores o peatones, derivadas de problemas de tráfico como congestiones vehiculares en horas pico, factores que hacen expresar ira o enojo de quienes se ven involucrados en ellos. La conducción arriesgada se define como el comportamiento temerario que las personas manifiestan al conducir; y la distracción al conducir se define como la desviación de la atención por parte de un individuo al conducir. En el presente estudio se realizaron los análisis de fiabilidad mediante la consistencia interna alfa de Cronbach, brindando los siguientes índices: en conducción agresiva (ítems 8, 10, 12, 14, 16, 18, 20, 22), .85; en conducción arriesgada (ítems 7, 9 , $11,13,15,17,19,21,23,24,25,26,27)$, .83; en distracción al conducir (ítems 1, 2, 3, 4, 5, 6), .79, sugiriendo estos resultados que la prueba se ajusta adecuadamente para medir estas variables en conductores salvadoreños.

\section{Resultados}

En el presente análisis de las dimensiones psicosociales y del comportamiento agresivo al conducir, en función de si sufrió un accidente de tránsito en los últimos seis meses, 
se aplicó la prueba t-Student para muestras independientes y se determinó la d de Cohen o tamaño del efecto mediante la calculadora de la Universidad de Colorado (EE. UU.). Al contrastar el estrés general en función de si sufrió un accidente de tránsito en los últimos seis meses, se encontró que existen diferencias estadísticas significativas entre los que sí lo sufrieron y los que no ( $t_{937}=3.60 ; \mathrm{p}<.001, \mathrm{~d}=.34$ ), presentando el grupo que lo experimentó un mayor efecto significativo de estrés general que los que no lo sufrieron. En bienestar psicológico, en función de si sufrió un accidente de tránsito en los últimos seis meses, se encontró que existen diferencias significativas entre los que lo sufrieron $y$ los que no ( $\mathrm{t}_{981}=3.92 ; \mathrm{p}<.001, \mathrm{~d}=.37$ ), presentando el grupo que lo sufrió mayor efecto significativo de falta de bienestar psicológico que los que no lo sufrieron. Al analizar la dimensión ansiedad, en función de si sufrió un accidente de tránsito en los últimos seis meses, se encontró que existen diferencias significativas entre los que lo sufrieron y los que no ( $\mathrm{t}_{976}=2.92 ; \mathrm{p}=.004, \mathrm{~d}=.26$ ), presentando el grupo que lo sufrió mayor efecto significativo de ansiedad que el grupo que no lo sufrió. En el análisis de la dimensión expresión verbal agresiva, en función de si sufrió un accidente de tránsito en los últimos seis meses, se encontró que existen diferencias estadísticas significativas entre quienes si lo sufrieron y los que no ( ${ }_{959}=3.12 ; p=.002$, $\mathrm{d}=.30$ ), presentando el grupo que lo sufrió mayor efecto significativo de expresión verbal agresiva que el grupo que no lo sufrió. Al analizar la dimensión uso del vehículo para expresar agresividad al conducir, en función de si experimentó un accidente de tránsito en los últimos seis meses, se encontró que existen diferencias significativas entre el grupo que lo experimentó que el grupo que no lo sufrió ( $t_{974}$ $=2.36 ; p=.018, d=.21$ ), presentando el grupo que lo experimentó mayor efecto significativo de uso del vehículo para expresar agresividad que el grupo que no lo experimentó. Al analizar la dimensión comportamiento agresivo al conducir, en función de si experimentó un accidente de tránsito en los últimos seis meses, se encontró que existen diferencias significativas entre los que lo experimentaron y los que no ( $t_{761}=3.12 ; p=.002, d=.31$ ), presentando el grupo que lo experimentó mayor efecto significativo de comportamiento agresivo al conducir que el grupo que no lo experimentó. Estos resultados confirman empíricamente que existe una correlación significativa entre las dimensiones psicosociales y el cometimiento de accidentes de tránsito. De igual forma, las dimensiones del comportamiento agresivo al conducir están correlacionadas con el cometimiento de accidentes de tránsito de los conductores salvadoreños (ver tabla 1).

Tabla 1. Diferencia de medias (M) y desviación típica (DT) de los factores psicosociales y del comportamiento agresivo al conducir en los conductores salvadoreños en función de si experimentó un accidente de tránsito en los últimos seis meses

\begin{tabular}{|c|c|c|c|c|c|c|c|}
\hline Dimensiones & & $\mathrm{n}$ & $\mathbf{M}$ & DT & $\mathbf{T}$ & $\mathbf{P}$ & $\begin{array}{l}\text { D de } \\
\text { Cohen }\end{array}$ \\
\hline \multirow[t]{2}{*}{ Bienestar laboral } & Sí & 116 & 26.20 & 6.15 & -2.21 & .027 & 0.21 \\
\hline & No & 787 & 27.49 & 5.84 & & & \\
\hline \multirow[t]{2}{*}{ Estrés general } & Sí & 112 & 25.58 & 6.10 & 3.60 & .000 & 0.34 \\
\hline & No & 827 & 23.60 & 5.40 & & & \\
\hline \multirow[t]{2}{*}{ Bienestar psicológico } & Sí & 121 & 9.46 & 2.61 & 3.92 & .000 & 0.37 \\
\hline & No & 862 & 8.52 & 2.46 & & & \\
\hline \multirow[t]{2}{*}{ Funcionamiento social } & Sí & 122 & 8.68 & 2.96 & 4.71 & .000 & 0.41 \\
\hline & No & 864 & 7.57 & 2.35 & & & \\
\hline \multirow[t]{2}{*}{ Salud mental } & Sí & 120 & 19.10 & 5.55 & 4.83 & .000 & 0.43 \\
\hline & No & 846 & 16.90 & 4.53 & & & \\
\hline \multirow[t]{2}{*}{ Ansiedad } & Sí & 120 & 15.39 & 4.18 & 2.92 & .004 & 0.26 \\
\hline & No & 858 & 14.34 & 3.64 & & & \\
\hline
\end{tabular}




\begin{tabular}{|c|c|c|c|c|c|c|c|}
\hline \multirow[t]{2}{*}{ Depresión } & Sí & 121 & 13.15 & 3.63 & 3.06 & .002 & 0.28 \\
\hline & No & 860 & 12.19 & 3.19 & & & \\
\hline \multirow[t]{2}{*}{ Expresión verbal agresiva } & Sí & 121 & 17.78 & 4.95 & 3.12 & .002 & 0.30 \\
\hline & No & 840 & 16.29 & 4.93 & & & \\
\hline \multirow[t]{2}{*}{ Expresión física agresiva } & Sí & 100 & 11.93 & 3.62 & 2.25 & .025 & 0.19 \\
\hline & No & 717 & 11.32 & 2.36 & & & \\
\hline \multirow[t]{2}{*}{ Uso del vehículo para expresar agresividad } & Sí & 120 & 15.18 & 4.53 & 2.36 & .018 & 0.21 \\
\hline & No & 856 & 14.28 & 3.80 & & & \\
\hline \multirow[t]{2}{*}{ Expresión adaptativa- constructiva } & Sí & 117 & 35.70 & 9.04 & -2.49 & .013 & 0.24 \\
\hline & No & 823 & 37.90 & 8.90 & & & \\
\hline \multirow[t]{2}{*}{ Conducción arriesgada } & Sí & 116 & 18.95 & 5.60 & 4.16 & .000 & 0.37 \\
\hline & No & 848 & 17.04 & 4.48 & & & \\
\hline \multirow[t]{2}{*}{ Conducción agresiva } & Sí & 122 & 10.47 & 3.73 & 3.54 & .000 & 0.31 \\
\hline & No & 859 & 9.42 & 2.96 & & & \\
\hline \multirow[t]{2}{*}{ Distracción al conducir } & Sí & 120 & 10.08 & 2.95 & 3.35 & .001 & 0.32 \\
\hline & No & 868 & 9.13 & 2.89 & & & \\
\hline \multirow[t]{2}{*}{ Comportamiento agresivo al conducir } & Sí & 95 & 35.05 & 9.02 & 3.12 & .002 & 0.31 \\
\hline & No & 668 & 32.41 & 7.55 & & & \\
\hline
\end{tabular}

Para establecer y determinar si existen correlaciones entre las dimensiones psicosociales y comportamiento agresivo al conducir, se aplicó la correlación de Pearson (r), encontrándose correlaciones estadísticamente significativas que van desde pequeñas y moderadas hasta grandes, tanto positivas como negativas. En este sentido, se tiene que existe correlación significativa entre bienestar laboral y bienestar psicológico $(r=-.302)$, entre estrés general y ansiedad $(r$ $=.694)$, entre estrés general y conducción arriesgada $(r=$ $.412)$, entre estrés general y conducción agresiva $(r=.411)$, entre bienestar psicológico y ansiedad $(r=.617)$, entre bienestar psicológico y expresión verbal agresiva $(r=.366)$, entre bienestar psicológico y comportamiento agresivo al conducir $(r=$.363). También se encontraron correlaciones significativas entre pequeñas, moderadas y altas en algunas dimensiones psicológicas y del comportamiento agresivo al conducir. En este sentido, se tiene que existe una correlación significativa entre salud mental y uso del vehículo para expresar agresividad $(r=.370)$, entre salud mental y comportamiento agresivo $(r=.373)$, entre ansiedad y depresión $(r=.657)$, entre ansiedad y comportamiento agresivo al conducir $(r=.417)$, entre depresión y uso del vehículo para expresar agresividad $(r=.381)$, entre el uso del vehículo para expresar agresividad y conducción agresiva $(r=.703)$, entre conducción arriesgada y comportamiento agresivo al conducir $(r=.685)$, entre conducción agresiva y comportamiento agresivo al conducir $(r=.788)$ y entre distracción al conducir y comportamiento agresivo $(r=.409)$ [ver tabla 2]. 


\begin{tabular}{|c|c|c|c|c|c|c|c|c|c|c|c|c|c|c|}
\hline U & & & & & & & & & & & & & & 菜 \\
\hline 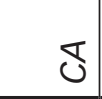 & & & & & & & & & & & & & $\stackrel{*}{\stackrel{*}{f}}$ & 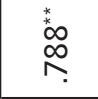 \\
\hline$\frac{\mathbb{c}}{\overleftarrow{S}}$ & & & & & & & & & & & & $\stackrel{*}{\stackrel{*}{\Sigma}}$ & $\begin{array}{l}\text { * } \\
\text { ஸे } \\
\text { மீ }\end{array}$ & $\begin{array}{l}* \\
\stackrel{*}{*} \\
\infty \\
0 \\
0\end{array}$ \\
\hline$\underset{\mathbb{4}}{\stackrel{\mathbb{x}}{*}}$ & & & & & & & & & & & $\frac{\text { * }}{\text { * }_{i}^{\circ}}$ & $\stackrel{*}{\stackrel{*}{*}}$ & $\underset{i}{\check{J}}$ & 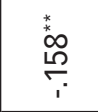 \\
\hline$\stackrel{\overleftarrow{J}}{3}$ & & & & & & & & & & & $\stackrel{\text { * }}{\text { * }}$ & 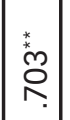 & 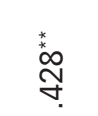 & ֻั. \\
\hline 㐍 & & & & & & & & & & 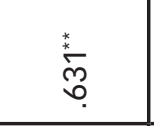 & 莣 & 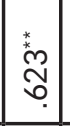 & సָ & 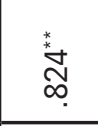 \\
\hline$\underset{w}{\mathbb{3}}$ & & & & & & & & & $\stackrel{*}{ \pm}$ & స్. & 。' & $\mid \begin{array}{l}* \\
\stackrel{*}{\sim} \\
\stackrel{N}{\lessgtr}\end{array}$ & $\stackrel{*}{*} \stackrel{*}{\stackrel{*}{*}}$ & $\stackrel{*}{*}$ \\
\hline 岀 & & & & & & & & 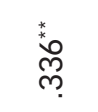 & $\stackrel{*}{\stackrel{*}{\text { จ }}}$ & 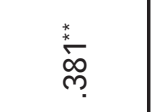 & 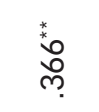 & $\left|\begin{array}{c}* \\
\stackrel{*}{n} \\
\hat{m} \\
\end{array}\right|$ & مِ & 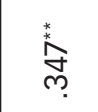 \\
\hline$\underset{\mathbf{T}}{Z}$ & & & & & & & 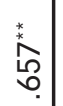 & $\begin{array}{l}\text { *. } \\
\text { స్ } \\
\text { m. }\end{array}$ & શั & 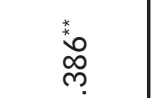 & 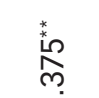 & $\mid \begin{array}{l}* \\
0 \\
0 \\
m\end{array}$ & 莣 & $\stackrel{*}{\stackrel{*}{8}}$ \\
\hline$\sum_{i s}$ & & & & & & 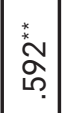 & $\frac{*}{\infty}$ & $\stackrel{*}{\stackrel{*}{N}}$ & ণิ & 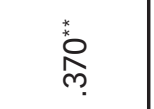 & $\begin{array}{l}\text { *a } \\
\text { o } \\
\text { m. }\end{array}$ & $\mid$\begin{tabular}{l}
$*$ \\
\multirow{2}{*}{} \\
m.
\end{tabular} & $\stackrel{\text { * }}{\text { : }}$ & $\stackrel{\text { }}{\stackrel{*}{m}}$ \\
\hline$\tilde{L}$ & & & & & $\stackrel{*}{\sigma}$ & 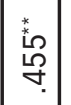 & 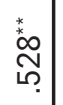 & থָ & $\stackrel{*}{\stackrel{*}{\circ}}$ & 㐘 & $\stackrel{*}{\stackrel{*}{N}}$ & $\mid$\begin{tabular}{l}
$*$ \\
\multirow{\alpha}{\sigma}{} \\
$\dot{\sigma}$
\end{tabular} & $\stackrel{*}{N}$ & $\begin{array}{l}\text { * } \\
\stackrel{*}{\circ}\end{array}$ \\
\hline$\frac{0}{00}$ & & & & $\begin{array}{l}\frac{*}{*} \\
0 \\
0\end{array}$ & 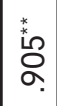 & $\frac{*}{\stackrel{*}{*}}$ & 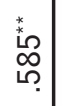 & $\begin{array}{l}\text { º } \\
\text { లి }\end{array}$ & $\stackrel{*}{\stackrel{*}{\stackrel{*}{N}}}$ & 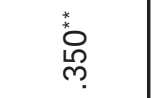 & $\begin{array}{l}\text { * } \\
\text { o } \\
0 \\
\text { m. }\end{array}$ & $\mid$\begin{tabular}{l}
$*$ \\
\multirow{2}{*}{} \\
m̧.
\end{tabular} & ํ. & 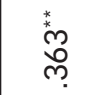 \\
\hline 岀 & & & 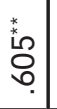 & 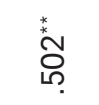 & $\frac{*}{5}$ & 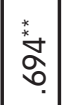 & 寺 & 今. & ণ & 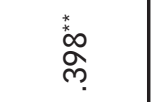 & $\stackrel{*}{\stackrel{*}{\succcurlyeq}}$ & 荌 & 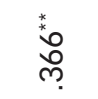 & $\frac{*}{\partial}$ \\
\hline $\overrightarrow{0}$ & & 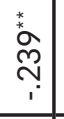 & 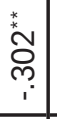 & $\stackrel{*}{\stackrel{*}{m}}$ & \begin{tabular}{|c|} 
\\
0 \\
0 \\
0 \\
0 \\
$\vdots$ \\
\end{tabular} & 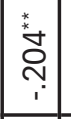 & 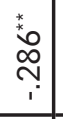 & $\underset{i}{\stackrel{*}{*}}$ & 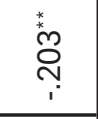 & $\begin{array}{l}{ }_{*}^{*} \\
\stackrel{\infty}{\infty} \\
\stackrel{1}{1}\end{array}$ & $\stackrel{*}{\underset{*}{\sim}}$ & \begin{tabular}{|c|c}
$*$ \\
$*$ \\
0 \\
0 \\
\hdashline \\
\end{tabular} & \begin{tabular}{l}
$*$ \\
\multirow{2}{*}{} \\
$\stackrel{2}{0}$ \\
$\stackrel{0}{0}$
\end{tabular} & $\underset{i}{\stackrel{*}{*}}$ \\
\hline 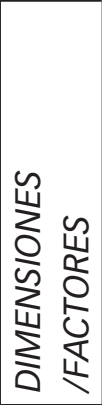 & 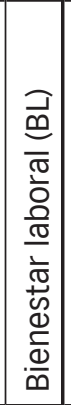 & 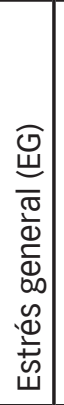 & 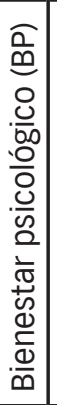 & 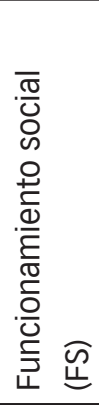 & 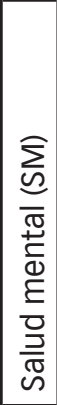 & \begin{tabular}{|l|}
2 \\
2 \\
$\frac{1}{5}$ \\
$\frac{0}{0}$ \\
$\frac{\pi}{0}$ \\
$\frac{0}{0}$ \\
$\frac{2}{4}$
\end{tabular} & 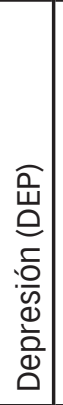 & 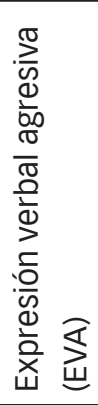 & 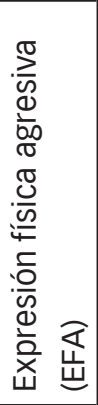 & 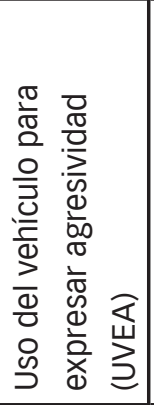 & 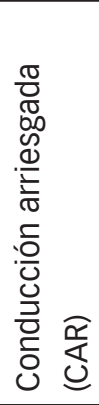 & 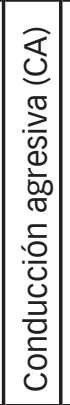 & 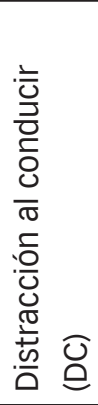 & 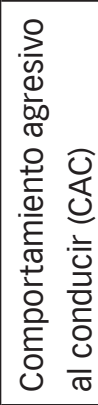 \\
\hline
\end{tabular}




\section{Discusión}

Discutir los factores psicosociales, en el contexto de la violencia del tráfico, es fundamental para comprender la esencia de algunos comportamientos de las personas. Al referirse a los factores psicosociales, de manera espontánea surge la idea de la salud, debido a que frecuentemente el término está asociado a la salud. Sin embargo, hay que aclarar que el término factores psicosociales es utilizado en diferentes circunstancias para explicar y comprender los determinantes de múltiples motivaciones, acciones, conductas, comportamientos del ser humano; usualmente se habla de factores psicosociales de riesgo de violencia, de la salud y del entorno laboral. En el presente estudio, los factores psicosociales de riesgo se refieren a aquellas dimensiones psicológicas y sociales que tienen como base la personalidad, el comportamiento agresivo al conducir y la salud, y que en la mayoría de veces son de alto riesgo en el cometimiento de accidentes de tránsito al conducir. Estos datos están en sintonía con los factores psicosociales de riesgo, que expresan que son probables de daño a la salud, tanto física como psicológica, resultando negativos (Cox, \& Griffiths, 1996). Son factores de estrés que pueden alterar y desequilibrar los recursos y las capacidades de la persona para manejar y responder al flujo de la actividad derivada del trabajo (Taris, \& Kompier, 2005). Los resultados del estudio evidencian que existe una correlación significativa de las dimensiones psicosociales, como el bienestar laboral, estrés general, bienestar psicológico, ansiedad, depresión y problemas de salud mental, con haber experimentado un accidente de tránsito en los últimos seis meses. Galovski y Blanchard (2002) demostraron que los conductores agresivos presentan mayores niveles de estrés general y ansiedad.

En el comportamiento agresivo al conducir, se tienen diferentes variables vinculadas con la conducta agresiva de los conductores salvadoreños, entre estas se tienen las siguientes: expresión verbal agresiva, expresión física agresiva, uso del vehículo para expresar agresividad, conducción arriesgada y distracción al conducir. Los conductores que se ven involucrados en altercados con otros conductores reportan más choques y más agresiones de tráfico que el resto de conductores (Hemenway \& Solnick, 1993). Los hallazgos evidencian que son significativas las dimensiones del comportamiento agresivo al conducir con el cometimiento de accidentes de tránsito. También se puede observar que, tanto en los distintos factores psicosociales como en los de comportamiento agresivo al conducir, tie- nen efectos pequeños significativos en la accidentabilidad de los conductores salvadoreños. En este sentido, se recomienda buscar mejorar las dimensiones psicosociales y del comportamiento agresivo para eliminar y disminuir la tasa de fallecidos y lesionados en accidentes de tránsito por cada 100 mil habitantes en El Salvador.

\section{Referencias}

Archer, J.; Kilpatrick, G. \& Bramwell, R. (1995). Comparison of two aggression inventories. Aggressive Behavior, 21, pp. 371-380.

Beck, A.T. (1970). Depression. Causes and treatment. Philadelphia. University of Pennsylvania Press.

Benavides, F.; Gimeno, D.; Benach, J.; Martínez, J.M.; Jarque, S., \& Berra, A. (2002). "Descripción de los factores de riesgo psicosocial en cuatro empresas". Gac Sanit, 16(3), pp. 222-229.

Bettencourt, B.A. \& Miller, N. (1996). "Gender differences in aggression as a function of provocation: A metaanalysis". Psychological Bulletin, 119, pp. 422-447.

Cooper, C.L. (1998). Theories of Organizational Stress. Oxford: Oxford University Press.

Cooper, C.L.; Dewe, Ph., \& O'Driscoll, M.P.(2001). Organizational Stress. Thousand Oaks: Sage Publications.

Cox, T. \& Griffiths, A.J. (1996). The assessment of psychosocial hazards at work. En M. J. Schabracq, J. A. M. Winnubst, \& C. L. Cooper (Eds.), Handbook of Work and Health Psychology. Chichester: Wiley and Sons; pp.127-146.

Deffenbacher, J.L.; Huff, M.E.; Lynch, R.S.; Oetting, E.R. \& Salvatore, N.E. (2000). "Characteristics and treatment of high anger drivers". Journal of Counselling Psychology, 47(1), pp. 5-12.

Deffenbacher, J.L.; Lynch, R.S.; Oetting, E.R., \& Swaim, R.C. (2002). "The Driving Anger Expression Inventory: A measure of how people express their anger on the road". Behaviour Research and Therapy, 40(6), 717-737.

Deffenbacher, J.L.; Lynch, R.S.; Oetting, E.R., \& Yingling, D.A. (2001). "Driving anger: Correlates and a test of statetrait theory". Personality and Individual Differences, 31(8), pp. 1321-1331.

Fondo de Prevención Vial (2003). Políticas para mejorar la seguridad vial en América Latina y el Caribe. Bogotá.

Galovski, T. \& Blanchard, E.B. (2001). "Characteristics of aggressive drivers with and without intermittent explosive disorder". Behaviour Research and Therapy, 40, pp. 1157-1168. 
Galovski, T. \& Blanchard, E.B. (2002). "The psychophysiology of aggressive drivers: Comparison to non-aggressive drivers and pre -to post- treatment change following a cognitive-behavioural treatment". Behaviour Research and Therapy, 41, pp. 1055-1067.

García-Pérez, E.M.; Magaz-Lago, A.; García-Campuzano, R.; Sandín-Iñigo, M.V. \& Ceregido-Senín, S. (2011). Escala Magallanes de Estrés. Grupo ALBOR-COHS, España.

Goldberg, D.P., \& Blackwell, B. (1970). "Psychiatric illness in general practice. A detailed study using a new method of case identification". British Medical Journal, 1, pp. 439-443.

Goldberg, D.; Bridges, K.; Duncan-Jones, P., \& Grayson, D. (1988). "Detecting anxiety and depression in general medical settings". British Medical Journal, 297, pp. 897-899.

Hemenway, D. \& Solnick, S.J. (1993), "Fuzzy Dice, Dream Cars and Indecent Gestures: Correlates of Driver Behavior?". Accident Analysis and Prevention, 25(2), pp. 161-170, and (1994) "Author's Rejoinder". Accident Analysis and Prevention, 26, pp. 128-129.

Herrero-Fernández, D.; Fonseca-Baeza, S. \& Pla-Sancho, S. (2014). "Estructura factorial del Driving Log en una muestra española". Revista de psicología, 32(1) pp. 67-90.

Hobfoll, S.E. (1989). Conservation of Ressources. A new attempt of conceptualizing stress. American Psychologist, 44(3), 513-524.

Junger, M.; West, R. \& Timman, R. (2005). "Crime and risky behavior in traffic: An example of cross-situational consistency". Journal of Research in Crime and Delinquency, 38, pp. 439-459.

Kalimo, R.; El-Batawi, M., \& Cooper, C. L. (OMS, 1988). LOS factores psicosociales en el trabajo y su relación con la salud. Ginebra.

Krahé, B. \& Fenske, I. (2002) "Predicting aggressive driving behavior: The role of macho personality, age, and power of car". Aggr. Behav. 2, pp. 21-29.

Lajunen, T.; Parker, D., \& Stradling, S.G. (1998). "Dimensions of driver anger, aggressive and highway code violations and their mediation by safety orientation". Transportation Research Part F, 1, pp. 107-121.

Lawton, R. \& Nutter, A. (2002). "A comparison of reported levels and expression of anger in everyday and driving situations". British Journal of Psychology, 93, pp. 407-423.

Lazarus, R. \& Folkman, S. (1986). Estrés y Procesos cognitivos. Barcelona: Martinez Roca.
Miguel-Tobal, J.J. (1996). La ansiedad. Madrid: Santillana.

Miles. D.E. \& Johnson, G.L. (2003). "Aggressive driving behaviors: Are there psychological and attitudinal predictors". Transportation Research Part, 6, pp. 147-161.

Ministerio de Salud (2016). "Informe de costos de salud en atención a víctimas de accidentes de tránsito". San Salvador, El Salvador.

Minztberg, H. (1993). Structure in fives: designing effective organizations. Englewood Cliff, N.J.: Prentice Hall.

Montero, I. \& León, O. (2007). “Sistema de clasificación del método en los informes de investigación en psicología". International Journal of Clinical and Health Psychology, 7(3), pp. 847-862.

Montón, C.; Pérez-Echevarría, M.J.; Campos, R.; GarcíaCampayo, J.; Lobo, A., \& Gzemmp (1993). "Escalas de ansiedad y depresión de Goldberg. Una guía de entrevista eficaz para la detección del malestar psíquico". Atención primaria, 12, pp. 345-349.

Okasha, A. (2005). "Globalization and mental health: a WPA perspective". World Psychiatry, 4(1), pp. 1-2.

Organización Internacional de Trabajo (1986). Factores psicosociales en el trabajo: reconocimiento y control. Ginebra.

Organización Mundial de la Salud (2015). "Informe sobre la situación mundial de seguridad vial".

Parry, M.H. (1968). Aggression on the road. Nueva York: Tavistock.

Policía Nacional Civil (2007). "Informe de estadísticas de siniestralidad de tránsito". San Salvador, El Salvador.

Policía Nacional Civil (2013). "Informe de estadísticas de víctimas de accidentes de tránsito". San Salvador, El Salvador.

Ruales, J. (OPS/OMS, 2014). "Estadísticas de los accidentes de tráfico en El Salvador". San Salvador, El Salvador.

Selye, H. (1975). Tensión sin angustia. Madrid: Guadarrama.

Taris, T. \& Kompier, M. (2005). "Job demands, job control and learning behavior: Review and research agenda". En Antoniou, A. \& Cooper, C. (Eds.) Research companion to Organizational Health Psychology. Cheltenham: Edward Elgar Publishing LTD; pp. 32-150.

Ulleberg, P. (2001). "Personality subtypes of young drivers. Relationship to risk-taking preferences, accident involvement, and response to a traffic safety campaing". Transportation Research Part, 4, pp. 279-297.

Ward, N.J., Waterman, M.G. \& Joint, M. (1998). "Rage and violence in driver aggression". En Grayson, G. (Ed.), Behavioural research in road safety VIII. Crowthorne: TRL. 